pag

Business School

WORKING PAPER SERIES

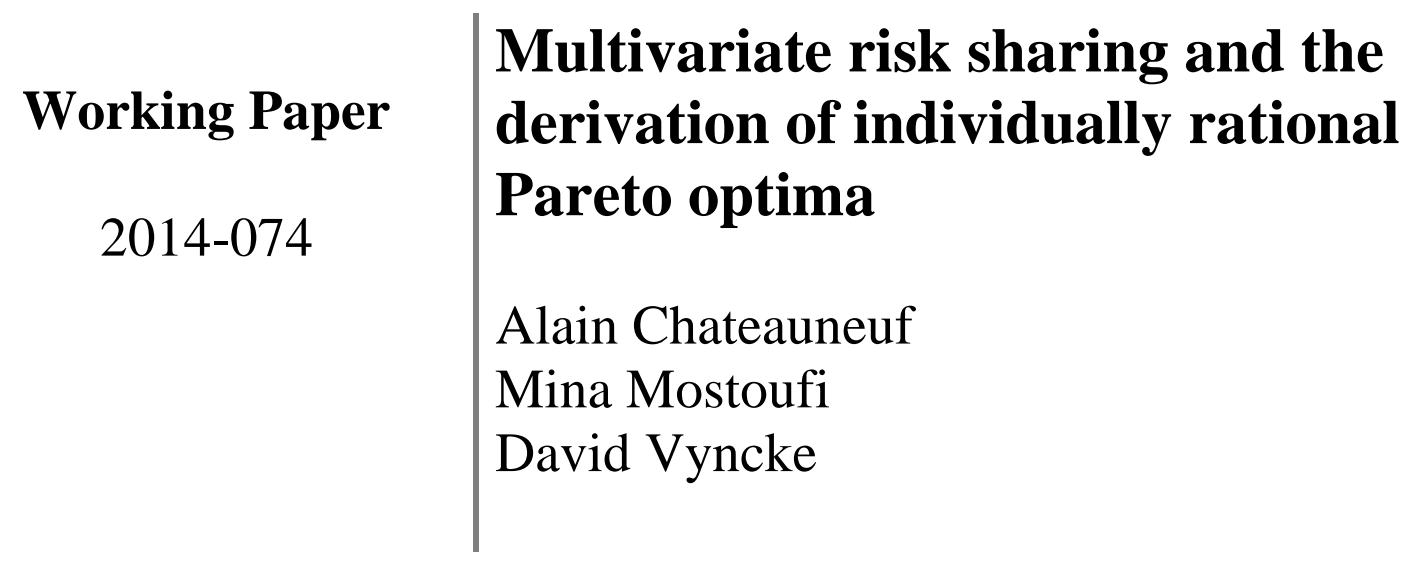

http://www.ipag.fr/fr/accueil/la-recherche/publications-WP.html

IPAG Business School

184, Boulevard Saint-Germain

75006 Paris

France 


\title{
Multivariate risk sharing and the derivation of individually rational Pareto optima
}

\author{
Alain Chateauneuf ${ }^{\mathrm{a}, *}$, Mina Mostoufi ${ }^{\mathrm{b}}$, David Vyncke ${ }^{\mathrm{c}}$ \\ ${ }^{a} I P A G$ Business School and PSE-Université de Paris I \\ ${ }^{b}$ Paris School of Economics and Université de Paris I \\ ${ }^{c}$ Ghent University
}

\begin{abstract}
Considering that a natural way of sharing risks in insurance companies is to require risk by risk Pareto optimality, we offer in case of strong risk aversion, a simple computable method for deriving all Pareto optima. More importantly all Individually Rational Pareto optima can be computed according to our method.
\end{abstract}

Keywords: Multivariate risk sharing, Comonotonicity, Individually rational Pareto optima

\section{Introduction}

In a seminal paper, Landsberger and Meilijson (1994) extended a central result of risk sharing established by Borch (1962) in the particular case of risk averse expected utility (EU) decision makers (DM). Namely Landsberger and Meilijson (1994) proved that for strong risk averse decision makers, efficient risk-sharing allocations still coincide with comonotone allocations.

Recently Carlier et al. (2012) generalized the comonotone dominance principle as well as the equivalence between efficiency and comonotonicity to the multidimensional case.

However, since Carlier et al. (2012) assumed that the agents have preferences agreeing with the multidimensional concave order, it turns out that their generalization of comonotonicity is far from being intuitive. Hence, their characterization of Pareto optima may appear as being somewhat awkward.

*alain.chateauneuf@univ-paris1.fr

January 17, 2014 
The goal of this paper is to use a simple, natural and meaningful definition of multidimensional risk aversion, namely component by component strong risk aversion.

This allows to characterize Pareto optimal allocations as the component by component comonotone allocations.

The main novelty provided by this work is to offer a complete characterization of Pareto optima, by extensively taking advantage of the polytope structure of these Pareto optima. Furthermore it is shown that this strategy also allows to easily describe the entire convex set of individually rational Pareto optima, under the mild assumption that the underlying probability information(we just consider a finite set of states of nature) consists of rational probabilities. This is not a too restrictive assumption since any probabilistic information can indeed be approximated as far as needed by such rational probabilities. The paper is organized as follows. Section 2 presents the framework and recalls some definitions. Section 3 deals with the characterization of Pareto optimal risk sharing, while section 4 offers a description of all individually rational Pareto optima. Finally, section 5 concludes the paper.

\section{Framework and Definitions}

Consider, for the purpose of illustration, $n$ insurance companies, $i=$ $1, \ldots, n$, each holds at date zero, $p$ portfolios of insurance of type $k=1, \ldots, p$ leading at date one to future stochastic wealth $X_{i}^{k}:\left(S, 2^{S}, P\right) \rightarrow \mathbb{R}_{+}$, where $S=\left(s_{1}, \ldots, s_{j}, \ldots, s_{m}\right)$ is the finite space of the sets of nature, and $P$ the probability on $2^{S}$ is given and satisfies $P\left(s_{j}\right)=p_{j}>0 \quad \forall j$.

Let $w_{i}=\left(w_{i}^{1}, \ldots, w_{i}^{k}, \ldots, w_{i}^{p}\right)$ be the initial endowment of insurance $i$ with respect to each portfolio of type $k$, i.e. each future wealth in each state with respect to premia and reimbursements related to type $k$. Denote $w^{k}=\sum_{i=1}^{n} w_{i}^{k}$.

By definition, $X$ is a feasible allocation if $X=\left(X_{1}, \ldots, X_{i}, \ldots, X_{n}\right)$ with $X_{i} \in\left(\mathbb{R}_{+}^{m}\right)^{p} \forall i=1, \ldots, n$ and $\sum_{i=1}^{n} X_{i}^{k}=w^{k} \quad \forall k \in \llbracket 1, p \rrbracket$.

Let us now recall that if $X$ and $Y$ are bounded real random variables, $X$ dominates $Y$ by the second order stochastic dominance i.e. $X$ is considered as less risky than $Y$ denoted by $X \succeq_{S S D} Y$ if $\int_{0}^{p} F_{X}^{-1}(t) d t \geq \int_{0}^{p} F_{Y}^{-1}(t) d t$ 
$\forall p \in \llbracket 0,1 \rrbracket$ where $F^{-1}$ is the usual quantile function.

Moreover $X \succ_{S S D} Y$ i.e. $X$ strictly dominates $Y$ for the second order stochastic dominance if furthermore $\int_{0}^{p_{0}} F_{X}^{-1}(t) d t>\int_{0}^{p_{0}} F_{Y}^{-1}(t) d t$ for some $p_{0} \in(0,1]$.

We assume that each agent $i$ has preferences $\succeq_{i}$ associated with the component by component second order stochastic dominance that is for $X_{i}=$ $\left(X_{i}^{1}, \ldots, X_{i}^{k}, \ldots, X_{i}^{p}\right) \in\left(\mathbb{R}_{+}^{m}\right)^{p}$ and $Y_{i}=\left(Y_{i}^{1}, \ldots, Y_{i}^{k}, \ldots, Y_{i}^{p}\right) \in\left(\mathbb{R}_{+}^{m}\right)^{p}$ then if $X_{i}^{k} \succeq_{S S D} Y_{i}^{k} \forall k \in \llbracket 1, p \rrbracket$ one has $X_{i}$ weakly preferred to $Y_{i}$ i.e. $X_{i} \succeq_{i} Y_{i}$ and if furthermore there exist $k_{0} \in \llbracket 1, p \rrbracket$ such that $X_{i}^{k_{0}} \succ_{S S D} Y_{i}^{k_{0}}$ then $X_{i}$ is strictly preferred to $Y_{i}$ i.e. $X_{i} \succ_{i} Y_{i}$.

From the above assumptions it turns out that:

\section{Definition 1}

$X=\left(X_{1}, \ldots, X_{i}, \ldots, X_{n}\right) \in\left(\mathbb{R}^{m}\right)^{p \times n}$ is Pareto optimal if $\forall k \in \llbracket 1, p \rrbracket$ $\left(X_{1}^{k}, \ldots, X_{i}^{k}, \ldots, X_{n}^{k}\right)$ is Pareto optimal in the usual sense for the univariate case with respect to the second order stochastic dominance i.e. for $k$ given:

$X_{i}^{k} \in \mathbb{R}_{+}^{m} \forall i, \sum_{i=1}^{n} X_{i}^{k}=w^{k}$ and there does not exist $Y=\left(Y_{1}^{k}, \ldots, Y_{i}^{k}, \ldots, Y_{n}^{k}\right)$, $Y_{i}^{k} \in \mathbb{R}_{+}^{m} \forall i, \sum_{i=1}^{n} Y_{i}^{k}=w^{k}$, such that $Y_{i}^{k} \succeq_{S S D} X_{i}^{k} \forall i$ and $Y_{i_{0}}^{k} \succ_{S S D} X_{i_{0}}^{k}$ for some $i_{0}$.

\section{Definition 2}

$X=\left(X_{1}, \ldots, X_{i}, \ldots, X_{n}\right) \in\left(\mathbb{R}^{m}\right)^{p \times n}$ is an individually rational Pareto optimum if $X$ is Pareto optimal and individually rational i.e. $\forall i, k X_{i}^{k} \succeq_{S S D} w_{i}^{k}$.

\section{Deriving all Pareto optima}

From Definition 1 it turns out that the $p$-dimensional case reduces to $p$ one dimensional situations. So we just have to deal with the following situation:

$X=\left(X_{1}, \ldots, X_{i}, \ldots, X_{n}\right) X_{i}:\left(S, 2^{S}, P\right) \rightarrow \mathbb{R}_{+}, w \in \mathbb{R}_{+}^{m}$ given. In subsection 3.1 for the sake of completeness we just propose what we hope to be a very simple, direct and complete proof of the well-known characterization of Pareto optimal allocations in terms of comonotonicity. 


\subsection{Pareto optima in the one-dimensional case}

\section{Definition 3}

An allocation $X=\left(X_{1}, \ldots, X_{i}, \ldots, X_{n}\right)$ is comonotone if,

$\forall\left(i, i^{\prime}\right) \in \llbracket 1, n \rrbracket^{2} \quad\left(X_{i}(s)-X_{i}(t)\right)\left(X_{i^{\prime}}(s)-X_{i^{\prime}}(t)\right) \geq 0 \quad \forall(s, t) \in S^{2}$.

We intend to retrieve, in a simple way, the well-known following theorem, which is implicit in Landsberger and Meilijson (Landsberger and Meilijson 1994).

\section{Theorem 1}

The set of Pareto optimal allocations coincide with the set of comonotone allocations.

The proof will result from the following two lemmas.

\section{Lemma 1}

Any Pareto optimal allocation is comonotone.

Proof: We just sketch the proof given in Chateauneuf et al. (2000). It is enough to show that any non-comonotone allocation $X=\left(X_{1}, \ldots, X_{i}, \ldots, X_{n}\right)$ can be improved to a new allocation $X^{\prime}=\left(X_{1}^{\prime}, \ldots, X_{i}^{\prime}, \ldots, X_{n}^{\prime}\right)$ which is mutually beneficial for all agents and strictly beneficial for at least one.

Let us assume, without loss of generality, that comonotonicity is not satisfied for $X_{1}, X_{2}$ and for $s_{1}, s_{2}$. Let $X_{1}\left(s_{1}\right)=x_{1}, X_{1}\left(s_{2}\right)=x_{2}, X_{2}\left(s_{1}\right)=y_{1}$, $X_{2}\left(s_{2}\right)=y_{2}$ and assume without loss of generality that $x_{1}+y_{1} \leq x_{2}+y_{2}$, $x_{1}>x_{2}$ and $y_{1}<y_{2}$. Let us modify $\left(x_{1}, x_{2}\right)$ to $\left(x_{1}^{\prime}, x_{2}^{\prime}\right)$ and $\left(y_{1}, y_{2}\right)$ to $\left(y_{1}^{\prime}, y_{2}^{\prime}\right)$ where $x_{1}^{\prime}=x_{2}^{\prime}=\frac{p_{1} x_{1}+p_{2} x_{2}}{p_{1}+p_{2}}, y_{1}^{\prime}=x_{1}+y_{1}-x_{1}^{\prime}$ and $y_{2}^{\prime}=x_{2}+y_{2}-x_{2}^{\prime}$. so $X=\left(X_{1}, \ldots, X_{i}, \ldots, X_{n}\right)$ has been modified to $X^{\prime}=\left(X_{1}^{\prime}, X_{2}^{\prime}, X_{3}^{\prime}, \ldots, X_{n}^{\prime}\right)$ where $X_{i}=X_{i} \quad \forall i=3, \ldots, n$.

It is then straightforward to see that we obtain a new allocation $X^{\prime}$ and that $X_{i}^{\prime}$ is strictly less risky than $X_{i}$ for $i=1,2$ since $E\left(u\left(X_{i}^{\prime}\right)\right)>E\left(u\left(X_{i}\right)\right)$ for any strictly concave utility function $u$, which completes the proof.

Lemma 2

Any comonotone allocation is Pareto optimal. 
Proof: Let $X=\left(X_{1}, \ldots, X_{i}, \ldots, X_{n}\right)$ be a comonotone allocation. We just intend to show that it is impossible that a feasible allocation $Y=$ $\left(Y_{1}, \ldots, Y_{i}, \ldots, Y_{n}\right)$ strictly dominates $X$. Without loss of generality, we assume that $Y_{1} \succ_{S S D} X_{1}$ i.e. there exists $p_{0} \in(0,1]$ such that:

$\int_{0}^{p_{0}} F_{Y_{1}}^{-1}(t) d t>\int_{0}^{p_{0}} F_{X_{1}}^{-1}(t) d t$

and,

$\int_{0}^{p} F_{Y_{1}}^{-1}(t) d t \geq \int_{0}^{p} F_{X_{1}}^{-1}(t) d t$ and $\forall p \in[0,1]$.

Moreover $\int_{0}^{p} F_{Y_{i}}^{-1}(t) d t \geq \int_{0}^{p} F_{X_{i}}^{-1}(t) d t \forall i \forall p \in[0,1]$.

Hence we get,

$$
\sum_{i=1}^{n} \int_{0}^{p_{0}} F_{Y_{i}}^{-1}(t) d t>\sum_{i=1}^{n} \int_{0}^{p_{0}} F_{X_{i}}^{-1}(t) d t
$$

Let us now show that,

$$
\sum_{i=1}^{n} \int_{0}^{p_{0}} F_{Y_{i}}^{-1}(t) d t \leq \int_{0}^{p_{0}} F_{\sum_{i=1}^{n} Y_{i}}^{-1}(t) d t
$$

Recall that TVAR is sub-additive see Denuit and Dhaene (2012), i.e. for any random variable $\left.Z, \operatorname{TVAR}(Z, p)=\frac{1}{1-p} \int_{p}^{1} F_{Z}^{-1}(t)\right) d t$, where $p \in[0,1)$ is such that for any random variables $T$ and $Z$ one gets:

$\operatorname{TVAR}(Z+T, p) \leq \operatorname{TVAR}(Z, p)+T \operatorname{VAR}(T, p)$

From $\left.\left.E(Z)=\int_{0}^{1} F_{Z}^{-1}(t)\right) d t, E(T)=\int_{0}^{1} F_{T}^{-1}(t)\right) d t$ and indeed $E(Z+T)=$ $E(Z)+E(T)$ it is then straightforward to obtain:

$$
\int_{0}^{p_{0}} F_{Z+T}^{-1}(t) d t \geq \int_{0}^{p_{0}} F_{Z}^{-1}(t) d t+\int_{0}^{p_{0}} F_{T}^{-1}(t) d t
$$


And therefore by induction one gets (2). Combining (1) and (2) we obtain:

$$
\sum_{i=1}^{n} \int_{0}^{p_{0}} F_{X_{i}}^{-1}(t) d t<\int_{0}^{p_{0}} F_{\sum_{i=1}^{n} Y_{i}}^{-1}(t) d t
$$

But $\sum_{i=1}^{n} X_{i}=w=\sum_{i=1}^{n} Y_{i}$ hence $F_{w}^{-1}=F_{\sum_{i=1}^{n} X_{i}}^{-1}=F_{\sum_{i=1}^{n} Y_{i}}^{-1}$,

moreover since $X$ is comonotone $F_{\sum_{i=1}^{n} X_{i}}^{-1}=\sum_{i=1}^{n} F_{X_{i}}^{-1}$ a.e. (almost everywhere) thus (3) implies:

$\int_{0}^{p_{0}} F_{w}^{-1}(t) d t<\int_{0}^{p_{0}} F_{w}^{-1}(t) d t$ a contradiction, which completes the proof of lemma 2 and henceforth of Theorem 1.

\subsection{Deriving all Pareto optima}

We intend now to show that the set of Pareto optima is a polytope. Therefore by implementing the vertex identification algorithm as can be found in MATLAB (2010), one can easily obtain all Pareto optima. Let us start by a preliminary lemma.

\section{Lemma 3}

Let $w\left(s_{j}\right)=w_{j}$. Then after possibly relabeling, if needed, the indices in such a way that $w_{1} \leq \ldots \leq w_{j} \leq \ldots \leq w_{m}$, one gets: If $\left(X_{i}\right)_{i=1, \ldots, n}$ is an allocation, then the two following properties are equivalent;

(i) $\quad\left(X_{i}\right)_{i=1, \ldots, n}$ is comonotone.

(ii) $\quad X_{i}(1) \leq \ldots \leq X_{i}(j) \leq \ldots \leq X_{i}(m) \forall i=1, \ldots, n$.

Proof:

$(i) \Longrightarrow(i i)$ : Let $w_{j} \leq w_{j+1}$ and assume there exists $i_{0}$ such that $X_{i_{0}}(j)>$ $X_{i_{0}}(j+1)$. Since $\left(X_{i}\right)_{i=1, \ldots, n}$ is comonotone, we have $X_{i}(j) \geq X_{i}(j+1) \forall i \neq i_{0}$. Summing up both sides of the inequality over $i=1, \ldots, n$ gives $\sum_{i=1}^{n} X_{i}(j)>$ $\sum_{i=1}^{n} X_{i}(j+1)$. Since $\left(X_{i}\right)_{i=1, \ldots, n}$ is a feasible allocation, the following relation $w_{j}>w_{j+1}$ is obtained, which is a contradiction. 
$(i i) \Longrightarrow(i)$ : Take $j \neq j^{\prime}$. From $(i i)$ we have:

$X_{i}(1) \leq \ldots \leq X_{i}(j) \leq \ldots \leq X_{i}(m) \forall i=1, \ldots, n$, therefore it is immediate that $\forall i \neq i^{\prime}\left(X_{i}(j)-X_{i}\left(j^{\prime}\right)\right)\left(X_{i^{\prime}}(j)-X_{i^{\prime}}\left(j^{\prime}\right)\right) \geq 0$, which means that the $X_{i}$ 's are pairwise comonotone.

\section{Theorem 2}

The set of Pareto optima is a polytope, hence it is the convex hull of its finitely many extreme points.

Proof: Since from Theorem 1, Pareto optima are comonotone allocations, it is straightforward from lemma 3 to see that the set $\mathcal{P}$ of Pareto optima is a nonempty compact convex subset $K$ of $\mathbb{R}^{n}$, defined as the intersection of some finite collection of closed half-spaces (that is the set of solutions of finitely many linear inequalities). Hence from proposition 3.2.1 in Florenzano et al. (2001) we conclude that $\mathcal{P}$ is a polytope, and from the well-known Krein-Milman theorem (see for instance, proposition 3.1.4 in Florenzano et al. (2001)) that $\mathcal{P}$ is the convex hull of its extreme points. Since $\mathcal{P}$ is a polytope, the number of these extreme points is finite.

\subsection{Two illustrating examples}

Here we present two examples to illustrate the procedure of the calculation of the extreme points; also we study the properties of the obtained vertices through these examples. In the first example, we chose the following values for the parameters introduced in the problem framework. We take $m=n=2$ and the initial vector of endowment $w=(2,4)$, which leads to the following constraints:

$\mathcal{P}=\left\{\left(x_{1}, x_{2}\right),\left(y_{1}, y_{2}\right)\right\}$ such that: $\left\{\begin{array}{l}-x_{1} \leq 0,-x_{2} \leq 0 \\ -y_{1} \leq 0,-y_{2} \leq 0 \\ x_{1}-x_{2} \leq 0, y_{1}-y_{2} \leq 0 \\ -x_{1}-y_{1} \leq-2,-x_{2}-y_{2} \leq-4 \\ x_{1}+y_{1} \leq 2, x_{2}+y_{2} \leq 4\end{array}\right.$

As it was discussed before, $\mathcal{P}$ is a polytope. Furthermore, $\mathcal{P}$ is the convex hull of the finite set of extreme points. For the sake of illustration, we obtain the extreme points of $\mathcal{P}$. First we construct the related system $\mathcal{S}$ of 
linear equations with the four unknown quantities $x_{1}, x_{2}, y_{1}$ and $y_{2}$ :

$$
\begin{aligned}
x_{1}+y_{1} & =2 \\
x_{2}+y_{2} & =4 \\
x_{1} & =0 \\
x_{2} & =0 \\
y_{1} & =0 \\
y_{2} & =0 \\
& =0 \\
x_{1}-x_{2} \quad & \\
y_{1}-y_{2} & =0
\end{aligned}
$$

Clearly any extreme point satisfies the endowment constraints (1) and (2). So building for instance upon Proposition 3.3.1 in Florenzano et al. (2001), we know that any extreme point is a solution of a subsystem of $\mathcal{S}$ including equalities (1) and (2), which is of rank 4, and which satisfies, the corresponding remaining inequalities of $\mathcal{P}$. First we pick equations (1), (2), (3) and (4), which leads to $\left(x_{1}=0, y_{1}=2\right)$ and $\left(x_{2}=0, y_{2}=4\right)$. Now we check the obtained result with the remaining inequality constraints, which proves that $\left(\left(x_{1}, x_{2}\right),\left(y_{1}, y_{2}\right)\right)$ is situated inside the polytope, therefore it is a feasible solution and can be regarded as one of the vertices of the polytope. We add $\left(x_{1}=0, y_{1}=2\right)$ and $\left(x_{2}=0, y_{2}=4\right)$ to the set of vertices and continue the algorithm for the remaining set of equations to identify all the extreme points. In this way four extreme points are identified as below:

$\left\{\left(\left(x_{1}, x_{2}\right),\left(y_{1}, y_{2}\right)\right)\right\}=\{((0,0),(2,4)),((2,4),(0,0)),((0,2),(2,2))((2,2),(0,2))\}$

As already mentioned in theorem 2, the set of Pareto optima can be defined as the convex hull of the identified extreme points. Hence, considering the extreme points obtained for this example, the set of Pareto optimal can be presented as below:

$$
\begin{array}{r}
\left\{\left(\left(x_{1}, x_{2}\right),\left(y_{1}, y_{2}\right)\right)\right\}=\text { lbrace }\left(2 \alpha_{2}+2 \alpha_{4}, 4 \alpha_{2}+2 \alpha_{3}+2 \alpha_{4}\right) \\
\left.\left(2 \alpha_{1}+2 \alpha_{3}, 4 \alpha_{1}+2 \alpha_{3}+2 \alpha_{4}\right)\right\}
\end{array}
$$


In the second example we consider the parameters, $m=3, n=2$ and $w=(3,5,3)$. The comonotonicity assumption requires reordering the initial endowment in an increasing order, which gives $w=(3,3,5)$. Now we can construct the system of equations and inequalities as described in the problem framework. The obtained feasible extreme points are as following:

$$
\begin{array}{r}
\left\{\left(\left(x_{1}, x_{2}, x_{3}\right),\left(y_{1}, y_{2}, y_{3}\right)\right)\right\}=\{((0,0,0),(3,3,5)),((3,3,5),(0,0,0)) \\
((0,0,2),(3,3,3))((3,3,3),(0,0,2))\}
\end{array}
$$

One important property of the obtained extreme points in this example and the previous one is the symmetry of results with respect to the replacing of $x_{i}$ by $y_{i}$. This property is due to the structure of equalities, imposed by endowment constraints. Hence if $\left(x_{i}, y_{i}\right)$ is an extreme point, $\left(y_{i}, x_{i}\right)$ is also an extreme point of the polytope ${ }^{1}$.

\section{Deriving all individually rational Pareto optima}

As for Pareto optima, it turns out from Definition 2 that the $p$-dimensional case reduces to $p$ one dimensional cases. Let $w_{i}:\left(S, 2^{S}, P\right) \rightarrow \mathbb{R}_{+}, i=1, \ldots, n$ be the initial endowments of the agents. We are looking for a Pareto optimum $X=\left(X_{1}, \ldots, X_{i}, \ldots, X_{n}\right)$ such that $X_{i} \succeq_{S S D} w_{i}, i=1, \ldots, n$.

Some preliminary lemmata:

\section{Lemma 4}

Any individually rational Pareto optimum (IRPO) $X_{i}$ is such that $E\left(X_{i}\right)=$ $E\left(w_{i}\right)$.

Proof: One has $\sum_{i=1}^{n} w_{i}=\sum_{i=1}^{n} X_{i}$ hence $\sum_{i=1}^{m} E\left(w_{i}\right)=\sum_{i=1}^{m} E\left(X_{i}\right)$ but $X_{i} \succeq_{S S D} w_{i}$ so $\int_{0}^{1} F_{X_{i}}^{-1}(t) d t \geq \int_{0}^{1} F_{w_{i}}^{-1}(t) d t$ i.e. $E\left(X_{i}\right) \geq E\left(w_{i}\right)$ which gives the result.

\footnotetext{
${ }^{1}$ Indeed implementing the vertex identification algorithm in MATLAB(2010) would give the set of Pareto optima in any case.
} 


\section{Lemma 5}

The set $\mathcal{P}_{I R}$ of individually rational Pareto optima is nonempty.

Proof: Landsberger and Meilijson (1994) nicely proved in their Proposition 1 (page 100) that every allocation is dominated by some comonotone allocation, therefore from Theorem 1 one can conclude that there exist at least one individually rational Pareto optimum.

\section{Remark 1}

Note that in the present paper, we intend to systematically derive all IRPO's at least for rational probabilities (which apparently in "real life" is not a severe limitation). Our result contrasts from the algorithms which can be found in the literature. Actually these algorithms propose a method to obtain only one IRPO (see e.g. Landsberger and Meilijson (1994) or Ludkovski and Rüschendorf (2008)), but not all IRPO's.

\section{Remark 2}

Note that even for a finite state space $S$, it is not easy to express the individually rational conditions $X_{i} \succeq_{S S D} w_{i}, i=1, \ldots, n$. Actually $X_{i} \succeq_{S S D} w_{i}$ is equivalent to

$$
\int_{0}^{p} F_{X_{i}}^{-1}(t) d t \geq \int_{0}^{p} F_{w_{i}}^{-1}(t) d t \quad \forall p \in(0,1)
$$

with equality if $p=1$, as noticed in Lemma 4 , but even if (6) has to be checked only for a finite number $p_{\ell} \in(0,1)$, in practice finding which $p_{\ell}$ must be chosen is a delicate task. In contrast, if each $p_{j}$ is a rational probability, let us say of the type $p_{j}=\frac{k_{j}}{q}$ where $k_{j}, q \in \mathbb{N}_{+}^{*}$, it is immediate that $X_{i} \succeq_{S S D} w_{i}$ iff $\int_{0}^{\frac{k}{q}} F_{X_{i}}^{-1}(t) d t \geq \int_{0}^{\frac{k}{q}} F_{w_{i}}^{-1}(t) d t \quad \forall k \in \llbracket 1, q \rrbracket$.

We then state Theorem 3, which is the main result of this paper.

\section{Theorem 3}

The set $\mathcal{P}_{I R}$ of individually rational Pareto optima is a polytope, hence the convex hull of its finitely many extreme points.

Proof: The proof is similar as the one of theorem 2 except that we have 
now to take into account the new "closed half-spaces" constraints $E\left(X_{i}\right)=$ $E\left(w_{i}\right) \forall i$ and $\int_{0}^{p_{\ell}} F_{X_{i}}^{-1}(t) d t \geq \int_{0}^{p_{\ell}} F_{w_{i}}^{-1}(t) d t \quad \forall i \forall p_{\ell}$. Note that IRPO's depend on the probability $P$ while $P O$ 's are independent of probability $P$.

\subsection{Two illustrating examples}

Example 1

Table 1:

\begin{tabular}{ccc}
\hline \hline State & $s_{1}$ & $s_{2}$ \\
\hline$p$ & $\frac{1}{2}$ & $\frac{1}{2}$ \\
\hline$w_{1}$ & 2 & 0 \\
$w_{2}$ & 0 & 4 \\
\hline$w$ & 2 & 4
\end{tabular}

One can imagine that 1 and 2 are agricultural producers and that $w_{1}, w_{2}$ represents the possible production of tomatoes during one year depending on the climate conditions $s_{1}$ and $s_{2}$. Note by the way that one could imagine that these agricultural producers produce also potatoes, so our definition of Pareto optima as Pareto optima separately with respect to tomatoes productions and potatoes productions would apparently make sense in such a situation.

Here we are looking for Pareto optima $X=\left(x_{1}, x_{2}\right)$ and $Y=\left(y_{1}, y_{2}\right)$. Clearly the Pareto optima $(X, Y)$ are characterized by:

Comonotonicity condition: $x_{1} \leq x_{2}$ and $y_{1} \leq y_{2}$

Dominance: $X \succeq_{S S D} w_{1}$ so $x_{1} \geq 0$ and $E(X)=E\left(w_{1}\right)$ i.e. $x_{1}+x_{2}=2$ $Y \succeq_{S S D} w_{2}$ so $y_{1} \geq 0$ and $E(Y)=E\left(w_{2}\right)$ i.e. $y_{1}+y_{2}=4$

Feasibility: $x_{1} \geq 0$ and $x_{2} \geq 0, y_{1} \geq 0$ and $y_{2} \geq 0$

$$
x_{1}+y_{1}=2, x_{2}+y_{2}=4
$$

Hence direct computations give that the extreme IRPO's are $((0,2),(2,2))$ and $((1,1),(1,3))$ so: 
$\mathcal{P}_{I R}=\left\{\left(\alpha_{2}, 2 \alpha_{1}+\alpha_{2}\right),\left(2 \alpha_{1}+\alpha_{2}, 2 \alpha_{1}+3 \alpha_{2}\right), \alpha_{1} \geq 0, \alpha_{2} \geq 0, \alpha_{1}+\alpha_{2}=1\right\}$

Example 2

Table 2:

\begin{tabular}{ccc}
\hline \hline State & $s_{1}$ & $s_{2}$ \\
\hline$p$ & $\frac{1}{4}$ & $\frac{3}{4}$ \\
\hline$w_{1}$ & 2 & 0 \\
$w_{2}$ & 0 & 4 \\
\hline$w$ & 2 & 4
\end{tabular}

Table 3: Converted to the uniform probability

\begin{tabular}{ccccc}
\hline \hline State & $s_{1}$ & $s_{21}$ & $s_{22}$ & $s_{23}$ \\
\hline$p$ & $\frac{1}{4}$ & $\frac{1}{4}$ & $\frac{1}{4}$ & $\frac{1}{4}$ \\
\hline$\hat{w}_{1}$ & 2 & 0 & 0 & 0 \\
$\hat{w}_{2}$ & 0 & 4 & 4 & 4 \\
\hline$\hat{w}$ & 2 & 4 & 4 & 4
\end{tabular}

Note that we can write the initial situation as in table 3 by taking into account that the true states that will occur are $s_{1}$ and $s_{2}$, and not $s_{1}, s_{21}, s_{22}$ and $s_{23}$. So Pareto optima will be $X=\left(x_{1}, x_{2}\right)$ and $Y=\left(y_{1}, y_{2}\right)$ or fictitious $\hat{X}=\left(x_{1}, x_{2}, x_{2}, x_{2}\right)$ and $\hat{Y}=\left(y_{1}, y_{2}, y_{2}, y_{2}\right)$, so the set $\mathcal{P}_{I R}$ will now satisfy the polytope property:

Comonotonicity condition: $x_{1} \leq x_{2}$ and $y_{1} \leq y_{2}$

Dominance: $X \succeq_{S S D} w_{1}, x_{1} \geq 0$ and $x_{1}+x_{2} \geq 0, x_{1}+2 x_{2} \geq 0$, $E(X)=E\left(w_{1}\right)$ i.e. $x_{1}+3 x_{2}=2$

$Y \succeq_{S S D} w_{2}, y_{1} \geq 0, y_{1}+y_{2} \geq 4, y_{1}+2 y_{2} \geq 8, E(Y)=E\left(w_{2}\right)$ i.e. $y_{1}+3 y_{2}=12$

Feasibility: $x_{1} \geq 0$ and $x_{2} \geq 0 y_{1} \geq 0$ and $y_{2} \geq 0$

$$
x_{1}+y_{1}=2, x_{2}+y_{2}=4
$$


Hence direct computation gives that the extreme IRPO's are $\left(\left(0, \frac{2}{3}\right),\left(2, \frac{10}{3}\right)\right)$ and $\left(\left(\frac{1}{2}, \frac{1}{2}\right),\left(\frac{3}{2}, \frac{7}{2}\right)\right)$ so:

$\mathcal{P}_{I R}=\left\{\left(\frac{1}{2} \alpha_{2}, \frac{2}{3} \alpha_{1}+\frac{1}{2} \alpha_{2}\right),\left(2 \alpha_{1}+\frac{3}{2} \alpha_{2}, \frac{10}{3} \alpha_{1}+\frac{7}{2} \alpha_{2}\right), \alpha_{1} \geq 0, \alpha_{2} \geq 0, \alpha_{1}+\alpha_{2}=1\right\}$

\section{Conclusion}

In this paper, in case of multiple risks, we did adopt the idea that a natural way for insurance companies to optimally share risks is risk by risk Pareto-optimality. Our framework is based upon the well-known results in the one dimensional case characterizing Pareto-optimality as comonotonicity in case of strong risk aversion. Two main results are obtained in this work. Due to the polytope structure of Pareto-optima and also of Individually Rational Pareto-optima, we offer a simple computable method. First for deriving all Pareto-optima and second -in the not severely restrictive case of rational probabilities- for deriving all Individually Rational Pareto-optima. The method merely consists in systematically obtaining the finitely many extreme points of the respective polytopes.

\section{References}

Borch, K., 1962. Equilibrium in a reinsurance market. Econometrica 30, 424-444.

Carlier, G., Dana, R.A., Galichon, A., 2012. Pareto efficiency for the concave order and multivariate comonotonicity. Journal of Economic Theory 147, 207-229.

Chateauneuf, A., Dana, R.A., Tallon, J.M., 2000. Optimal risk-sharing rules and equilibria with choquet-expected-utility. Journal of Mathematical Economics 34, 191-214.

Denuit, M., Dhaene, J., 2012. Convex order and comonotonic conditional mean risk sharing. Insurance: Mathematics and Economics 51, 265 - 270.

Florenzano, M., Le Van, C., Gourdel, P., 2001. Finite dimensional convexity and optimization. Springer New York. 
Landsberger, M., Meilijson, I., 1994. Co-monotone allocations, bickellehmann dispersion and the arrow-pratt measure of risk aversion. Annals of Operations Research 52, 97-106.

Ludkovski, M., Rüschendorf, L., 2008. On comonotonicity of pareto optimal risk sharing. Statistics \& Probability Letters 78, 1181-1188.

MATLAB, 2010. version 7.10.0 (R2010a). The MathWorks Inc., Natick, Massachusetts. 